\title{
Occurrence and potential human health risk of pharmaceutical residues in drinking water from Putrajaya (Malaysia)
}

\begin{abstract}
Occurrence of pharmaceutical residues in drinking water has been widely reported in countries that have registered steady economic growth. This can exert concerns among the general consumers, prompting them to explore the potential human health risks associated with continuous exposure to pharmaceuticals. However, such an occurrence is rarely reported in developing or under-developed countries. To give more contexts, this study looked at the presence of nine pharmaceutical residues in drinking water (amoxicillin, caffeine, chloramphenicol, ciprofloxacin, dexamethasone, diclofenac, nitrofurazone, sulfamethoxazole, and triclosan) at Putrajaya residential area in Malaysia. Additionally, the potential health risks associated with contaminated drinking water were investigated. This study has found the presence of pharmaceutical residue concentrations up to $0.38 \mathrm{ng} / \mathrm{L}$, with the highest concentration of caffeine $(0.38 \mathrm{ng} / \mathrm{L})$ and the lowest concentration of diclofenac $(0.14 \mathrm{ng} / \mathrm{L})$. In comparison, all the nine pharmaceutical residues were substantially lower than previously reported studies. In general, Hazard Quotient (HQ) values indicated that low potential health hazards were present for all age groups. Nevertheless, quantitative occurrences of pharmaceutical residues in drinking water will help guide future toxicological studies to examine other chronic effects, while canvassing for proper framework to look into the water risk management and regulation in Malaysia.
\end{abstract}

Keyword: Drinking water; Health risks; Occurrence; Pharmaceutical 